\title{
Invasive micropapillary component and its clinico-histopathological significance in patients with colorectal cancer
}

\author{
KATARZYNA JAKUBOWSKA, KATARZYNA GUZIŃSKA-USTYMOWICZ and ANNA PRYCZYNICZ \\ Department of General Pathomorphology, Medical University of Białystok, Białystok, 15-269 Podlaskie, Poland
}

Received July 13, 2015; Accepted April 29, 2016

DOI: $10.3892 / \mathrm{ol} .2016 .4717$

\begin{abstract}
Micropapillary components are located in distinct, empty spaces resembling lymphatic vessels and consist of clusters of cancer cells that adhere tightly to one another. These structures do not have a fibrovascular core. Invasive micropapillary components (IMPCs) exhibit reverse polarity, which results in a characteristic 'inside-out' structure. The aim of the present study was to examine the histological significance of the micropapillary component in colorectal carcinoma compared with conventional colorectal adenocarcinoma. Among 115 patients, 5 (4.3\%) were diagnosed with a micropapillary colorectal component based on hematoxylin and eosin staining and immunohistochemical analysis. To exclude identification of poorly-differentiated clusters of adenocarcinoma cells in the lymphatic vessels, immunohistochemical analysis with D2-40 was performed, and epithelial membrane antigen was used to confirm the specific 'inside-out' structure of IMPCs. IMPCs were observed to positively correlate with histopathological type $(\mathrm{P}=0.001)$ and tumor invasion in venous vessels $(\mathrm{P}=0.033)$. Furthermore, the presence and number of lymph node metastases was greater in IMPC cases compared with conventional carcinoma cases; however, these differences were not statistically significant $(\mathrm{P}=0.087$ and $\mathrm{P}=0.094$, respectively). In addition, IMPC cases were not significantly associated with the presence of inflammatory infiltrate in the invasive front of the tumor $(\mathrm{P}=0.098)$. Therefore, the present study indicates that the IMPC serves a histopathological and prognostic role in the diagnosis of colorectal cancer due to its aggressive and invasive behavior. IMPC is rare in cases of colorectal cancer and remains a great diagnostic challenge in pathomorphology with further detailed investigation required in the future.
\end{abstract}

Correspondence to: Mrs. Katarzyna Jakubowska, Department of General Pathomorphology, Medical University of Białystok, Jerzego Waszyngtona 13, Białystok, 15-269 Podlaskie, Poland

E-mail:kathian@wp.pl

Key words: invasive micropapillary carcinoma, colorectal cancer, micropapillary component

\section{Introduction}

IMPCs are located in distinct, empty spaces resembling small, extended lymphatic vessels and contain clusters of cancer cells that adhere tightly to one another (1-4). The clusters do not have a fibrovascular core $(3,5)$. The spaces are separated from one another by thin bands of fibrous tissue and form a structure comparable to a sponge. Nests may also form with fusiform-like cancer cells filling up single spaces (6,7), and are often observed in invasive tumor margins or less frequently in the tumor center (1,7-9). The micropapillary structure may constitute one of the morphological tumor components and coexist with other histological types of cancer, or it can be the only morphological feature $(3,5,8)$. However, cases of cancer that are formed of only micropapillary components are extremely rare (8). IMPC cells exhibit reverse polarity, which results in a characteristic 'inside-out' structure, for example, the basal surface of cells demonstrate properties typically observed in the apical region. Electron microscopy has previously confirmed that numerous microvilli line the outer surface of IMPC cells, which target their secretory activity towards the surrounding stroma. Furthermore, a limited amount of mucous secretion has been observed in spaces surrounding tumor cell nests $(10,11)$. These findings have been supported by immunohistochemistry using anti-mucin 1, cell surface associated (MUC1) antibodies, with results demonstrating that MUC1 is primarily located in the outer surface of epithelial cells in patients with IMPC compared with the color reaction located in the apical part of normal glandular cells (12). The reverse polarity of IMPC cells disturbs adhesion, conditions their malignancy and determines secretory properties. All the aforementioned morphological features are responsible for vascular and stromal invasion of tumor cells, promoting lymph node involvement and metastasis of cancer cells.

Micropapillary structures were first identified in invasive ductal breast cancer (13), and were also later identified in malignant tumors of the ovaries, urinary bladder, salivary glands and lungs (14-17). Previous studies have confirmed the presence of these structures in malignancies of the digestive system (9). In addition, it has been demonstrated that the presence of a micropapillary component in tumors indicates aggressiveness and poor clinical outcome (14-18). Therefore, the present study aimed to investigate the histological significance of micropapillary components in colorectal carcinoma compared with conventional colorectal adenocarcinoma. 

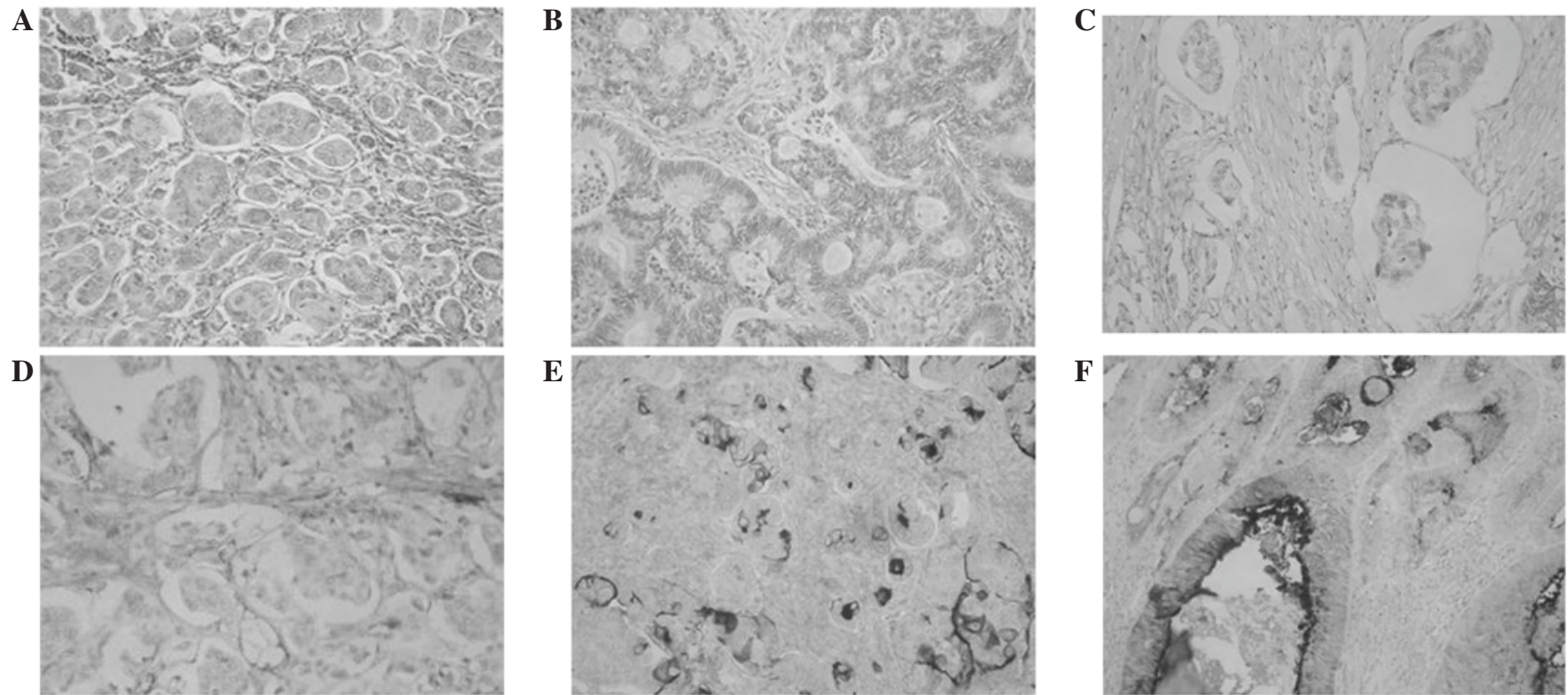

Figure 1. Characteristics of IMPC in patients with colorectal cancer. (A) IMPC is indicated by clusters of cancer cells without a fibrovascular core located in the invasive front of the tumor (staining, H\&E). (B) Morphological features of conventional adenocarcinoma (staining, H\&E). (C) A lack of D2-40 expression excluded lymphovascular invasion and confirmed the presence of IMPC, (D) in addition to confirming metastasis of adenocarcinoma cluster cells to lymphatic vessels. (E) Positive outer membrane expression of epithelial membrane antigen, which is characteristic for IMPC structures. (F) Cytoplasmic reactivity for EMA staining in conventional adenocarcinoma cells. Magnification, x200. IMPC, invasive micropapillary components; H\&E, hematoxylin and eosin.

\section{Materials and methods}

Materials. The study group consisted of 115 colorectal cancer patients, 53 women and 62 men, who had all been treated surgically in the Second Department of General and Gastroenterological Surgery, Medical University of Białystok (Białystok, Poland) between 2009 and 2013. The age of the patients ranged from 34-86 years old. All samples were pathologically diagnosed as colorectal cancer and diagnoses included the following information: Macroscopic tumor localization, histopathological type, anal invasion, primary tumor (pT) status, malignancy stage, lymph node involvement and metastases to distant organs according to Tumor-Node-Metastasis (TNM) classification guidelines (19). In addition, peritumoral inflammatory infiltrate and venous invasion were noted. Peritumoral inflammation was classified as follows: Absent, 0; mild, 1; moderate, 2; and marked, 3.

The present study was performed in accordance with the Declaration of Helsinki for Human Experimentation and was approved by the local Bioethics Committee (Nr R-I-002/406/2014). All participants provided written informed consent prior to examination.

Micropapillary component analysis. The micropapillary colorectal component was diagnosed according to 4- $\mu \mathrm{m}$ formalin-fixed and paraffin-embedded sections that underwent hematoxylin and eosin staining, and immunohistochemical analysis. To exclude poorly-differentiated clusters of adenocarcinoma cells in lymphovascular vessels, immunohistochemical staining was performed with an endothelial cell-specific monoclonal mouse anti-human podoplanin antibody (clone D2-40; \#05463645001; Roche Diagnostics, Warsaw, Poland; dilution, $0.12 \mu \mathrm{g} / \mathrm{ml}$ ). Reverse polarity, resulting in a characteristic 'inside-out' pattern of
IMPC, was identified following incubation with a monoclonal mouse antibody against epithelial membrane antigen (EMA) (E29 clone; \#05878900001; Roche Diagnostics; dilution, $0.54 \mu \mathrm{g} / \mathrm{ml}$ ). The reaction was performed using the Novocastra Novolink Polymer Detection system (Novocastra; Leica Biosystems, Milton Keynes, UK). A color reaction for peroxidase was developed with chromogene diaminobenzidine. Positive and negative controls were performed according to the manufacturer's protocols (Novocastra; Leica Biosystems). Counterstaining was performed with hematoxylin.

Statistical analysis. Statistical analysis was performed using STATISTICA 10.0 software (StatSoft, Inc., Tulsa, OK, USA). The $\chi^{2}$-quadrate coefficient test was used to analyze associations between parameters. $\mathrm{P}<0.05$ was considered to indicate a statistically significant difference. Cases with missing data for any variables were removed from the sample.

\section{Results}

Patient clinicopathological characteristics. Pathological analysis confirmed the diagnosis of colorectal cancer in all 115 cases and stage was determined according to the World Health Organization classification system (19). Out of 115 patients, adenocarcinoma type was diagnosed in 3 patients with IMPC and 99 patients with conventional carcinoma, whereas adenocarcinoma with a mucous component was diagnosed in 2 individuals with IMPC and 11 with conventional carcinoma. Tumors invaded into the anal canal in 4 cases of IMPC and 57 cases of conventional carcinoma. Tumors were classified as moderately-differentiated (G2) in 5 patients with IMPC and 104 patients with conventional carcinoma, and only 6 patients with conventional carcinoma were classified as having poorly-differentiated (G3) tumors. Only 1 patient 
with conventional carcinoma was staged as pT1, 6 patients as pT2, 100 patients as pT3 and 3 patients as pT4, whilst all patients with IMPC were staged as pT3. In addition, inflammatory infiltrate was absent in 1 patient and weak in 4 patients with IMPC, and was absent, weak, moderate and marked in 20, 47, 36 and 7 conventional carcinoma cases, respectively. The presence of venous invasion was observed in all patients with IMPC and in 58 patients with conventional carcinoma. At the time of the diagnosis, 4 out of 5 patients with IMPC and 51 out of 110 patients with conventional carcinoma presented with metastases at local lymph nodes, whereas only 28 out of 110 conventional carcinoma cases presented with metastases at distant organs.

Micropapillary component examination. Among the study group, 5 out of $115(4.3 \%)$ cases were diagnosed with a micropapillary colorectal component. Micropapillary components accounted for $2-10 \%$ of the tumor volume, and were situated in the center of the tumor mass in 2 cases, and at the invasive front in 3 (Fig. 1A). Conventional adenocarcinoma was observed in 95.7\% of CRC cases (Fig. 1B). Negative reactivity for podoplanin confirmed the presence of a micropapillary component (Fig. 1C), whereas positive reactivity for this protein was present in the endothelium (Fig. 1D). In patients with IMPC, positive EMA expression was observed on the outer membrane of epithelial cells (Fig.1E), whilst in normal glandular cells, EMA was expressed in luminal regions (Fig. 1F).

Associations between IMPC and selected clinicopathological parameters. The presence of IMPC was observed to positively correlate with histopathological type $(\mathrm{P}=0.001)$ and tumor invasion into venous vessels $(\mathrm{P}=0.033)$. Furthermore, the presence and number of lymph node metastases was greater in IMPC cases compared with conventional carcinoma cases, but the difference was not statistically significant $(\mathrm{P}=0.087$ and $\mathrm{P}=0.094$, respectively). IMPC demonstrated a negative association with the presence of peritumoral inflammatory infiltrate $(\mathrm{P}=0.098)$. No statistically significant differences were observed between IMPC and the remaining clinicopathological parameters, including age, gender, tumor localization, pT status, stage and distant organ metastasis. The results are presented in Table I.

\section{Discussion}

Micropapillary components are rare in forms of gastrointestinal cancer and typically constitute one of several histological types (6). In colon cancer, a micropapillary structure has been observed in $9-19 \%$ of all malignancies affecting the organ $(7,20,21)$. The present study identified only 5 patients with IMPC accounting for $4.3 \%$ of all study cases. The area occupied by the micropapillary component may be between $5-95 \%$ of the tumor volume and is most commonly located in the invasive front of the tumor $(3,6,7)$. Verdú et al (6) described the coexistence of an early cancer of the sigmoid with IMPC in a pedunculated polyp obtained during colonoscopy, the component being the major tumor morphological feature. Furthermore, Kondo et al (2) reported of a case of tubulovillous adenoma case in which a small area of IMPC developed. Lino-Silva (8) described a patient that presented with pure rectal IMPC, in which the micropapillary structure occupied $>95 \%$ of the lesion (8). In the current study, the micropapillary components accounted for $2-10 \%$ of the lesions and were located in the center of the tumor mass in 2 patients and in the invasive front in 3 cases. In accordance with previously reported cases, all IMPC cases in the present study coexisted with a moderately-differentiated form of cancer invading the subserosal layer through the muscular layer $(1,3,7,20,21)$.

Statistical analysis performed in the current study demonstrated that IMPCs accompany non-mucinous adenocarcinomas more frequently than mucinous carcinoma ( $\mathrm{P}=0.001)$. Similarly, Kim et al (20) described the presence of micropapillary components in a case of intestinal non-mucinous adenocarcinoma. These observations are also consistent with findings reported by Vendu et al (6), Haupt et al (7), Lino-Silva (8) and $\mathrm{Xu}$ et al (22), in which IMPC coexisted with adenocarcinoma of the colon. Colorectal adenocarcinomas consist of a phenotypically heterogenic population of cancer cells derived from glandular epithelium, which may be affected by epigenetic lesions and mutations (19). As a result, cancer cells acquire features of high-grade malignancy and manifest these in a specific morphological manner, for example, through the formation of micropapillary structures or mucus production capacity. This may explain the rare co-occurrence of IMPC and mucinous adenocarcinoma.

The presence of IMPC in patients with colorectal cancer has been previously demonstrated to positively correlate with lymph node invasion by cancer cells. Vendu et al (6) noted a that invasion of blood vessels was significantly more frequent in patients with IMPC compared with patients with conventional adenocarcinoma. However, differences have been reported between respective studies regarding the involvement of blood and lymphatic vessels in cases of IMPC in colorectal cancer. Vessels were invaded in the majority of all single colorectal cancer cases accompanied by IMPC described in the literature (1-3,18), whilst studies analyzing larger groups report that vascular involvement was noted in $40-45 \%$ of patients with colorectal cancer and $\operatorname{IMPC}(7,20)$. By contrast, Lino-Silva (8) and $\mathrm{Xu}$ et al (22) observed neoplastic cell emboli in the blood and lymphatic vessels in approximately one third of patients. These reports and the results of the present study suggest that IMPC in colorectal cancer may be responsible for high malignancy and cancer cell invasiveness. Furthermore, the present study observed a higher incidence of lymph node metastases in patients with IMPC, and according to the literature, lymph node involvement is estimated to occur in $63-100 \%$ of all cases $(6,17)$. Kim et al (20) confirmed the significance of intestinal cancer IMPC in pathomorphological diagnostics. The authors observed the presence of local lymph node involvement in 2 out of 3 patients with an IMPC tumor invading the submucosal membrane (pT1). These findings indicate the importance of early diagnosis through the analysis of biopsy and surgical specimens, as the lesion may determine whether the patient has a high risk of developing metastases. A number of studies reported that IMPC invasion was observed in up to $90 \%$ of resected lymph nodes and was the only histological component of the metastases that had formed $(3,5,20)$. The invasive properties of IMPC structures are associated with respective adhesion proteins: Outer membrane expression 
Table I. Associations between IMPC and non-IMPC and selected clinicopathological parameters.

\begin{tabular}{|c|c|c|c|c|}
\hline Parameter & IMPC & Non-IMPC & $\mathrm{R}$ & P-value \\
\hline Gender & & & -0.059 & 0.528 \\
\hline Female & 3 & 50 & & \\
\hline Male & 2 & 60 & & \\
\hline Age, years & & & 0.129 & 0.168 \\
\hline$<60$ & 0 & 31 & & \\
\hline$>60$ & 5 & 79 & & \\
\hline Localization & & & -0.090 & 0.336 \\
\hline Colon & 4 & 64 & & \\
\hline Rectum & 1 & 46 & & \\
\hline Histological type & & & 0.293 & $0.001^{\mathrm{a}}$ \\
\hline Non-mucinous & 3 & 64 & & \\
\hline Mucinous & 2 & 46 & & \\
\hline pT anal invasion & & & 0.195 & 0.153 \\
\hline Present & 4 & 57 & & \\
\hline Absent & 1 & 53 & & \\
\hline pT stage & & & 0.035 & 0.710 \\
\hline 1 & 0 & 1 & & \\
\hline 2 & 0 & 6 & & \\
\hline 3 & 5 & 100 & & \\
\hline 4 & 0 & 3 & & \\
\hline Malignancy stage & & & -0.045 & 0.628 \\
\hline G2 & 5 & 104 & & \\
\hline G3 & 0 & 6 & & \\
\hline Inflammation at invasive tumor front & & & -0.225 & 0.098 \\
\hline 0 & 1 & 20 & & \\
\hline 1 & 4 & 47 & & \\
\hline 2 & 0 & 36 & & \\
\hline 3 & 0 & 7 & & \\
\hline Venous invasion & & & 0.288 & $0.033^{\mathrm{a}}$ \\
\hline Present & 5 & 58 & & \\
\hline Absent & 0 & 51 & & \\
\hline Lymph node involvement & & & 0.233 & 0.087 \\
\hline Present & 4 & 51 & & \\
\hline Absent & 1 & 59 & & \\
\hline Lymph node status, no. & & & 0.228 & 0.094 \\
\hline$<5$ & 2 & 45 & & \\
\hline$>5$ & 3 & 6 & & \\
\hline Distant metastasis & & & -0.121 & 0.198 \\
\hline Present & 0 & 28 & & \\
\hline Absent & 5 & 82 & & \\
\hline
\end{tabular}

${ }^{\mathrm{a}} \mathrm{P}<0.05$. Data are presented as the number of cases. IMPC, invasive micropapillary component; $\mathrm{R}$, correlation coefficient; $\mathrm{pT}$, primary tumor.

of MUC1 was confirmed to indicate cancer cell adhesion disorders and induce disturbances in their association with the extracellular matrix (23). Furthermore, positive expression of E-cadherin was identified in the cytoplasm of cells with a micropapillary structure as compared with neoplastic glandular ducts and normal ducts (24).
The present study also observed that IMPC lesions in colorectal cancer were less frequently accompanied by peritumoral inflammatory infiltrate compared with conventional adenocarcinoma, although the differences were not significant. This association has never been confirmed in any previously reported IMPC colorectal cancer cases. It 
may be hypothesized that IMPC may induce suppression of the immune system, thus creating an optimal microenvironment for the primary tumor to grow and metastasize. In the digestive system, massive inflammatory infiltrate composed of neutrophilic granulocytes, which form clusters in the stroma and focal endothelial microabscesses, have only been identified in patients with pancreatic IMPC (24).

In conclusion, the associations identified by the present study indicate the highly aggressive and invasive nature of IMPC. Therefore, detection of these structures in the colon is histopathologically and prognostically significant. However, considering the relatively rare occurrence of IMPCs, these structures continue to present a diagnostic challenge for pathomorphologists, and require thorough analysis for an accurate diagnosis and prognosis.

\section{References}

1. Sonoo H, Kameyama M, Inatugi N, Nonomura A and Enomoto Y Pedunculated polyp of early sigmoid colon cancer with invasive micropapillary carcinoma. Jpn J Clin Oncol 39: 523-527, 2009.

2. Kondo T: Colon invasive micropapillary carcinoma arising in tubulovillous adenoma. Pol J Pathol 59: 183-185, 2008.

3. Sakamoto K, Watanabe M, De La Cruz C, Honda H, Ise H, Mitsui K, Namiki K, Mikami Y, Moriya T and Sasano H: Primary invasive micropapillary carcinoma of the colon. Histopathology 47: 479-484, 2005

4. Ushiku T, Matsusaka K, Iwasaki Y, Tateishi Y, Funata N, Seto Y and Fukayama M: Gastric carcinoma with invasive micropapillary pattern and its association with lymph node metastasis. Histopathology 59: 1081-1089, 2011.

5. Wen P, Xu Y, Frankel WL and Shen R: Invasive micropapillary carcinoma of the sigmoid colon: Distinct morphology and aggressive behavior. Int J Clin Exp Pathol 1: 457-460, 2008.

6. Verdú M, Román R, Calvo M, Rodón N, García B, González M, Vidal A and Puig X: Clinicopathological and molecular characterization of colorectal micropapillary carcinoma. Mod Pathol 24: 729-738, 2011.

7. Haupt B, Ro JY, Schwartz MR and Shen SS: Colorectal adenocarcinoma with micropapillary pattern and its association with lymph node metastasis. Mod Pathol 20: 729-733, 2007.

8. Lino-Silva LS: Pure micropapillary rectal carcinoma with CK 7 and CK20 coexpression and loss of CDX2 reactivity. Int J Morphol 30: 25-29, 2012.

9. Ohtsuki Y,KurodaN,YunokiS,MurakamiS,Mizukami Y,Okada Y, Iguchi M,Lee GH and Furihata M: Immunohistochemical analysis of invasive micropapillary carcinoma pattern in four cases of gastric cancer. Med Mol Morphol 46: 114-121, 2013.

10. Seidman JD and Kurman RJ: Subclassification of serous borderline tumors of the ovary into benign and malignant types. A clinicopathologic study of 65 advanced stage cases. Am J Surg Pathol 20: 1331-1345, 1996.
11. Luna-Moré S, Gonzalez B, Acedo C, Rodrigo I and Luna C: Invasive micropapillary carcinoma of the breast. A new special type of invasive mammary carcinoma. Pathol Res Pract 190: 668-674, 1994

12. Li YS, Kaneko M, Sakamoto DG, Takeshima Y and Inai K: The reversed apical pattern of MUC1 expression is characteristics of invasive micropapillary carcinoma of the breast. Breast Cancer 13: 58-63, 2006.

13. Siriaunkgul S and Tavassoli FA: Invasive micropapillary carcinoma of the breast. Mod Pathol 6: 660-662, 1993.

14. Amin MB, Ro JY, el-Sharkawy T, Lee KM, Troncoso P, Silva EG, Ordóñez NG and Ayala AG: Micropapillary variant of transitional cell carcinoma of the urinary bladder. Histologic pattern resembling ovarian papillary serous carcinoma. Am J Surg Pathol 18: 1224-1232, 1994.

15. Amin MB, Tamboli P, Merchant SH, Ordóñez NG, Ro J, Ayala AG and Ro JY: Micropapillary component in lung adenocarcinoma: A distinctive histologic feature with possible prognostic significance. Am J Surg Pathol 26: 358-364, 2002.

16. Nagao T, Gaffey TA, Visscher DW, Kay PA, Minato H, Serizawa H and Lewis JE: Invasive micropapillary salivary duct carcinoma: A distinct histologic variant with biologic significance. Am J Surg Pathol 28: 319-326, 2004.

17. Laurent I, Uzan C, Gouy S, Pautier P, Duvillard P and Morice P: Results after conservative treatment of serous borderline tumors of the ovary with a micropapillary pattern. Ann Surg Oncol 15: 3561-3566, 2008.

18. Kuroda N, Oonishi K, Ohara M, Hirouchi T, Mizuno K, Hayashi Y and Lee GH: Invasive micropapillary carcinoma of the colon: An immunohistochemical study. Med Mol Morphol 40: 226-230, 2007.

19. Hamilton SR, Rubio CA, Vogelstein B, Sobin LH, Kudo S, Fogt F, Riboli E, Winawer SJ, Nakamura S, Goldgar DE, et al: Tumours of the colon and rectum: Carcinoma of the colon and rectum. In: World Health Organization Classification of Tumours - Pathology and Genetics of Tumours of the Digestive System. Hamilton SR and Aaltonen LA (eds). IARC Press, Lyon, France, pp104-110, 2000.

20. Kim MJ, Hong SM, Jang SJ, Yu E, Kim JS, Kim KR, Gong G and Ro JY: Invasive colorectal micropapillary carcinoma: An aggressive variant of adenocarcinoma. Hum Pathol 37: 809-815, 2006.

21. Lino-Silva LS, Salcedo-Hernández RA and Caro-Sánchez CH: Colonic micropapillary carcinoma, a recently recognized subtype associated with histological adverse factors: Clinicopathological analysis of 15 cases. Colorectal Dis 14: e567-e572, 2012.

22. Xu F, Xu J, Lou Z, Di M, Wang F, Hu H and Lai M: Micropapillary component in colorectal carcinoma is associated with lymph node metastasis in T1 and T2 Stages and decreased survival time in TNM stages I and II. Am J Surg Pathol 33: 1287-1292, 2009.

23. Wesseling J, van der Valk SW, Vos HL, Sonnenberg A and Hilkens J: Episialin (MUC1) overexpression inhibits integrin-mediated cell adhesion to extracellular matrix components. J Cell Biol 129: 255-265, 1995.

24. Khayyata S, Basturk O and Adsay NV: Invasive micropapillary carcinomas of the ampullo-pancreatobiliary region and their association with tumor-infiltrating neutrophils. Mod Pathol 18: 1504-1511, 2005. 